\title{
Estudio de las poblaciones de cetáceos y detección de Zonas Especiales de Conservación para los cetáceos en el entorno marino de Jaizkibel y aguas adyacentes
}

\author{
Zetazeoen populazioen ikerketa eta Jaizkibelgo inguru itsastarrean \\ eta alboko uretan zetazeoentzat Kontserbazio Bereziko Eremuen detekzioa

\section{Cetacean population research and detection of Special Areas of Conservation for cetaceans in the marine environment of Jaizkibel and adjacent waters}

\author{
Enara Marcos-Ipiña ${ }^{1 *}$, Juan Manuel Salazar ${ }^{1,2}$, Renaud De Stephanis ${ }^{2}$ \\ ${ }^{1}$ EIBE, Baserritar etorbidea 6D 3ำ, 20080 Hondarribia. \\ ${ }^{2}$ CIRCE (Conservation Information and Research on Cetaceans), Cabeza de Manzaneda 3, Pelayo, 11390 Algeciras. \\ *Correspondencia: enara@eibeh.org
}

\section{RESUMEN}

Durante el periodo 2003-2010, se ha estudiado la diversidad, distribución y abundancia relativa de cetáceos en la costa vasca con el objetivo de detectar Zonas Especiales de Conservación para los cetáceos. Para ello se han realizado muestreos visuales a bordo de embarcación, mediante transectos aleatorios y en condiciones de esfuerzo óptimas. En total se han muestreado $13.770 \mathrm{~km}$, cubriendo un área total de $6.960 \mathrm{~km}^{2}$ de la costa vasca. Se ha invertido un mayor esfuerzo en los $40 \mathrm{~km}$ adyacentes a la costa guipuzcoana, donde se han identificado 12 especies de cetáceos. Los análisis sobre distribución temporal indican la presencia continua de 5 de ellas: el delfín mular Tursiops truncatus (Montagu, 1821), el delfín común Delphinus delphis (Linnaeus, 1758), el calderón común Globicephala melas (Traill, 1809), el delfín listado Stenella coeruleoalba (Meyen, 1833) y el zifio de Cuvier Ziphius cavirostris (Cuvier, 1823). Mediante la aplicación de modelos de análisis espacial se han identificado las principales áreas de acción para estas especies. El área de acción del delfín común y el delfín mular abarca el entorno marino inmediato a Jaizkibel, donde también se han detectado, de forma ocasional pero continua a lo largo de la serie temporal, tres especies de misticetos: el rorcual aliblanco Balaenoptera acutorostrata (Lacépède, 1804), la yubarta Megaptera novaeangliae (Borowski, 1781) y el rorcual común Balaenoptera physalus (Linnaeus, 1758). Destaca el interés del área para el delfín mular, debido a su alta densidad poblacional. Un estudio realizado a mayor escala espacial corrobora la importancia de este entorno marino para esta especie, cuya densidad poblacional es la mayor de toda la Demarcación Marina Noratlántica del norte de la Península Ibérica.

PALABRAS CLAVE: Abundancia relativa, distribución espacial y temporal, diversidad, Gipuzkoa.

\section{LABURPENA}

2003-2010 urteetan zehar, zetazeoen aniztasuna, dentsitatea, distribuzio espaziala eta tenporalaren azterketa etengabea gauzatu da Euskal Autonomia Erkidegoko uretan, bertan, zetazeoentzat Kontserbazio Bereziko Eremuak hantzemateko helburuarekin. Itsasontziz laginketa bisualak egin dira, trantsektu aleatorioaren metodologiaren bidez 13.770 km nabigatu direlarik, 6.960 km² ko azalera miatuz. Gipuzkoako kostaldeko lehen 40 kilometroetan ikerketa sakonagoa izan da, bertan 12 zetazeo espezieen presentzia baieztatu delarik. Distribuzio tenporalaren azterketaren arabera bost espezieren presentzia jarraia frogatu da; izurde haundia Tursiops truncatus (Montagu, 1821), izurde arrunta Delphinus delphis (Linnaeus, 1758), izurde pilotu hegaluzea Globicephala melas (Traill, 1809), izurde marraduna Stenella coeruleoalba (Meyen, 1833) eta Cuvier zifioak Ziphius cavirostris (Cuvier, 1823). Modelizazio espazialaren frogei esker, espezie hauen banaketa eremu printzipalak mugatu dira. Izurde haundiaren eta izurde arruntaren bizi eremua Jaizkibel ingurua barneratzen du, eremu hontan, maiztasun txikiarekin, baina etengabe ikerketa iraun duen denboran zehar, hiru miztizeto espezie ikustatu dira, zere hegatsuria Balaenoptera acutorostrata (Lacépède, 1804), zere arrunta Balaenoptera physalus (Linnaeus, 1758) eta xibarta balea Megaptera novaeangliae (Borowski, 1781). Izurde haundiaren populazioaren dentsitatearen arabera,bereziki garrantsitsua da azterturiko eremua. Azalera haundiago batean garaturiko ikerketa batek ondorio hau baieztatzen du, Penintsularen Iparraldeko Ipar Atlantikoko Demarkazio Itsastarrean espezie honen dentsitatea haundiena dela frogatuz.

GAKO-HITZAK: Gipuzkoa, dibertsitatea, distribuzio tenporala eta espaziala, dentsitatea, abundantzia.

\begin{abstract}
During the period 2003-2010, cetacean diversity, distribution and relative abundance have been continuously studied in waters of the Autonomous Community of the Basque Country, with the aim of detecting Special Areas for Conservation of cetaceans. Shipboard visual surveys have been carried out, by means of random transect methodology and with optimal effort conditions $13,770 \mathrm{~km}$ have been sailed, covering 6,960 $\mathrm{km}^{2}$ area. Higher survey effort was applied in the eastern part of the whole area, in the $40 \mathrm{~km}$ adjacent to coast of Gipuzkoa, where 12 cetacean species have been identified. Spatial and temporal distribution studies showed the continued presence of 5 cetacean species; bottlenose dolphin Tursiops truncatus (Montagu, 1821), common dolphin Delphinus delphis (Linnaeus, 1758), long-finned pilot whale Globicephala melas (Traill, 1809), striped dolphin Stenella coeruleoalba (Meyen, 1833) and Cuvier's beaked whale Ziphius cavirostris (Cuvier, 1823). Common dolphin and bottlenose dolphin home range covers the immediate marine environment of Jaizkibel, where minke whales Balaenoptera acutoros trata (Lacépède, 1804), humpback whale Megaptera novaeangliae (Borowski, 1781) and fin whales Balaenoptera physalus (Linnaeus, 1758) appear occasionally but constantly over the time series. The area has been proved to be important for bottlenose dolphins, due to its high population density. A study on a larger spatial scale corroborates the importance of this marine environment for the species, whose population density is the largest in the North Atlantic Marine Demarcation of the North Iberian Peninsula.
\end{abstract}

KEY WORDS: Gipuzkoa, diversity, spatial and temporal distribution, density, relative abundance. 


\section{INTRODUCCIÓN}

El Golfo de Bizkaia es conocido por su alta productividad y por ser una de las zonas con mayor diversidad de cetáceos en el Atlántico Nordeste (Hoyt 2005; Walker 2005a, 2005b). Como parte de este entorno marino, la costa vasca comparte muchas características ambientales con estas aguas, aunque posee aspectos biogeográficos que la hacen diferente (Borja, 2004) La compleja hidrología, los regímenes de sedimentación y la consecuente aportación de nutrientes favorecen la producción primaria en la que se basa una extensa red trófica. Por otro lado, en una estrecha franja de unos $36 \mathrm{~km}$, la plataforma continental, el talud y la fosa marina de Cap Breton, crean una gran variedad de nichos ecológicos, lo que permite el desarrollo de distintas comunidades biológicas y, por lo tanto, de una gran biodiversidad (Borja, 2004; De Casamajor, 2004; Valencia, 2004)

Especies como la sardina Sardina pichardus (Walbaum, 1792), la anchoa Engraulis encrasicolus (Linnaeus, 1758), la merluza Merluccius merluccius (Linnaeus, 1758), el verdel Scomber scombrus (Linnaeus, 1758), el congrio Conger conger (Linnaeus, 1758), el lirio Micromesistius poutassou (Risso, 1827), una gran variedad de cefalópodos (Arregi, 2004) y de eufásidos como Meganycthiphanes norvegica (M. Sars, 1857) (Nogeira, 2013) son presas potenciales para numerosas especies de cetáceos en aguas del Golfo de Vizcaya (Spitz et al., 2006, 2011; Anderwald et al., 2007; Santos et al., 2007; Lens et al., 2008; Read et al., 2008). Esta diversidad de presas puede explicar, en parte, la presencia de al menos 17 especies de cetáceos en la costa vasca (Marcos et al., 2005, 2013; Vázquez et al., 2005; Salazar et al., 2006, 2007).

Durante la última década, se ha realizado un esfuerzo por parte de distintas organizaciones (AMBAR, AZTI, CEMMA, GEFMA, EIBE) por estudiar estas especies de mamíferos marinos. En este trabajo se presentan los resultados obtenidos a partir de diversos estudios realizados durante el periodo 2003-2010 por la organización EIBE, con el objetivo de conocer la riqueza de especies, determinar la distribución temporal y espacial, calcular la densidad poblacional y abundancia absoluta de las distintas especies de cetáceos en el entorno marino de Jaizkibel y el área adyacente de la costa guipuzcoana.

\section{METODOLOGÍA \\ Marco de estudio}

Los resultados presentados en este trabajo se enmarcan dentro de dos proyectos principales, a saber: (1) "Estudio preliminar para realizar una propuesta de Lugares de Interés Comunitario (LIC) en el Litoral de la Comunidad Autónoma del País Vasco" (EIBE, 2010); (2) "Bases para el desarrollo de los planes de conservación de las especies de cetáceos protegidas en la Demarcación Marina Noratlántica (DMN)" (CEMMA, 2012). El objetivo de este úl- timo proyecto ha sido realizar una propuesta de planes de conservación para aquellas especies de cetáceos incluidas en el Catálogo Nacional de Especies Amenazadas (R.D 439/1990), además de contribuir a la revisión del estado de conservación de las especies de cetáceos incluidas en la categoría de "Vulnerable" presentes en la DMN.

\section{Área de estudio y protocolo de muestreo}

El área de estudio se extiende aproximadamente a lo largo de una franja de $40 \mathrm{~km}$ de ancho contigua a la costa guipuzcoana, que abarca un área total de 2.321 $\mathrm{km}^{2}$ (Fig. 1). Se han realizado sucesivos muestreos, durante el periodo que va desde Mar. de 2003 a Sep. de 2010. Mediante transectos aleatorios (11.353 km) se ha buscado cubrir los distintos pisos batimétricos que caracterizan el área de muestreo, a saber: la plataforma continental (0-200 $\mathrm{m}$ de profundidad), el talud continental (200-800 m) y la fosa oceánica (> 800 m). Durante estos transectos se registraron datos sobres las condiciones de esfuerzo de búsqueda, datos ambientales (estado de la mar, visibilidad, nubosidad, viento, temperatura) y datos sobre los avistamientos de cetáceos (especie, número de individuos observados, composición grupal, actividad, comportamiento), siguiendo los protocolos de metodología de la Sociedad Española de Cetáceos (SEC, 1999).

\section{Análisis de datos}

Para el análisis, únicamente se han empleado aquellos datos obtenidos durante los muestreos con esfuerzo de búsqueda óptimos. El esfuerzo de búsqueda se define como el número de kilómetros muestreados en condiciones de búsqueda adecuados (con el estado de la mar $\leq 3$ según la escala Douglas ${ }^{1}$ y buenas condiciones de visibilidad) y observadores en los puestos de vigilancia. En total, se han muestreado $10.988 \mathrm{~km}$ en condiciones de esfuerzo de búsqueda adecuadas. Así mismo, se han tenido en cuenta tan solo aquellos avistamientos en los que ha habido contacto (observación de un cetáceo) a menos de $100 \mathrm{~m}$ de distancia respecto del observador. No obstante, para la descripción de las especies presentes en el área adyacente a Jaizkibel, en el caso concreto del rorcual común Balaenoptera physalus (Linnaeus, 1758), también se han tenido en cuenta 3 avistamientos realizados sin registrar el esfuerzo de búsqueda. Así mismo, se han añadido avistamientos desde tierra y avistamientos oportunistas para describir la presencia y los movimientos de delfines comunes Delphinus delphis (Linnaeus, 1758) y delfines mulares Tursiops truncatus (Montahu, 1821) en la costa adyacente a Jaizkibel.

Para el análisis de la distribución temporal se ha calculado la Tasa de Encuentro (TE), que es el resultado de dividir el número de avistamientos de una especie determinada (Avs.) por el número de kilómetros muestreados 


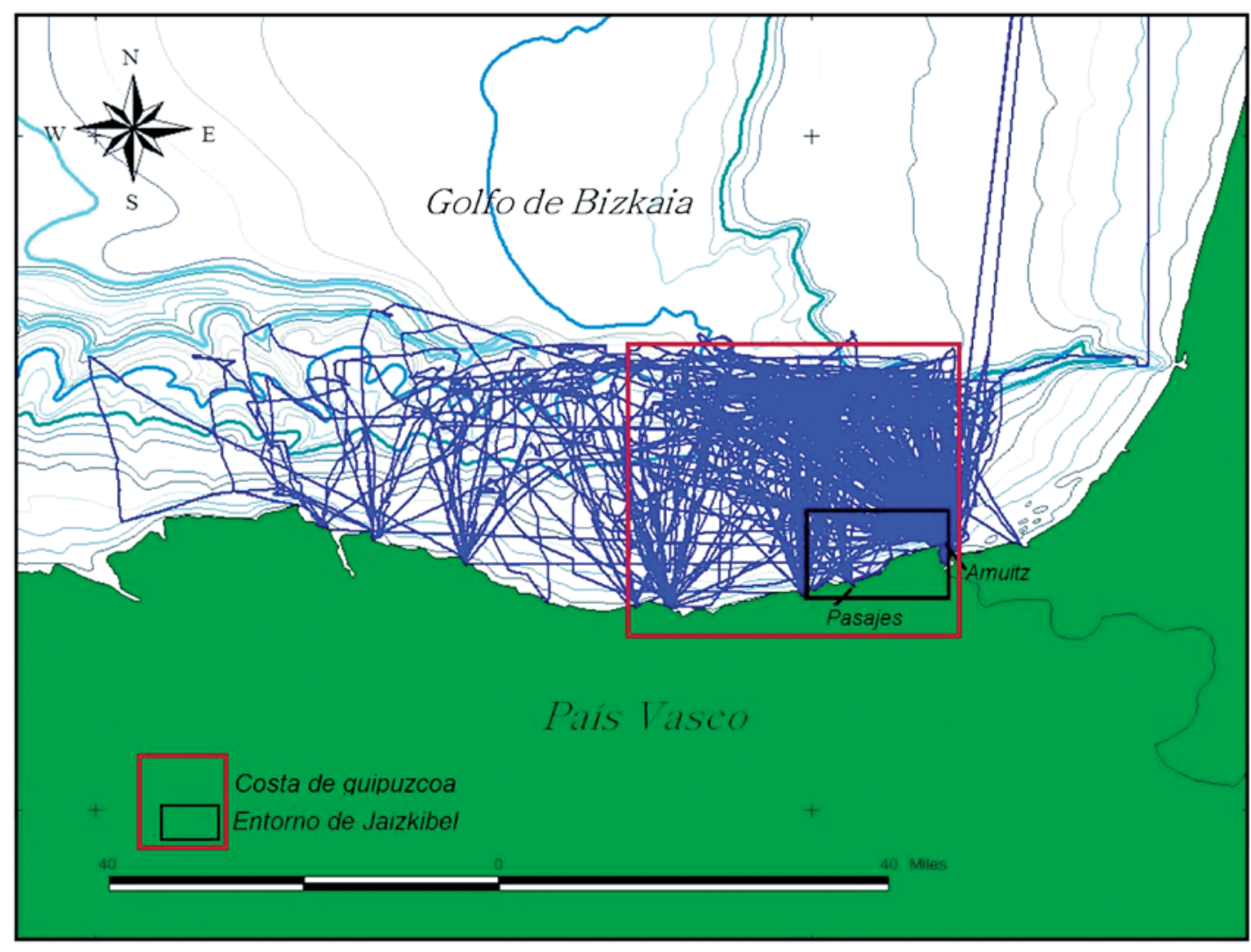

Fig. 1. - Área de estudio. Muestreos 2003-2010.

1. Irudia. Ikerketa eremua.Laginketak 2003-2010.

(Esf.), expresado en porcentaje: TE = (Avs./Esf.)x100. Las unidades temporales empleadas para el análisis son: otoño (Oct.-Dic.), invierno (Ene.-Mar.), primavera (Abr.-Jun.) y verano (Jul.-Sep.). Similarmente, las TE han sido utilizadas para determinar la abundancia relativa de las especies.

Se ha analizado la distribución espacial para las especies presentes a lo largo de toda la serie temporal, con el fin de definir el área de campeo para cada especie. Para este análisis espacial el área de estudio se ha dividido en cuadriculas (unidades de esfuerzo) con una resolución de 2 minutos de latitud por 2 de longitud. La distancia en kilómetros de búsqueda en cada cuadrícula se ha calculado mediante el sistema de información geográfica ArcView 3.2 de ESRI y su extensión Animal Movement (Hooge \& Eichenlaub, 2000). Para el análisis, únicamente se han tenido en cuenta aquellas celdas en las que se han recorrido un mínimo de $3 \mathrm{~km}$ con esfuerzo de búsqueda.

El análisis gráfico de la distribución espacial se ha realizado mediante análisis de polígonos de kernel fijos , el mejor estimador disponible para estimar el área de campeo (Worton, 1995; Seaman \& Powell, 1996; Seaman et al., 1999; Powell, 2000). Este estimador no paramétrico proporciona un modelo probabilístico para áreas de campeo que describe la cantidad relativa de tiempo que un animal pasa en un lugar mediante el cálculo del índice UD (Utilization Distribution). Los polígonos kernel fijos se calcularon a partir de la tasa de encuentro por especie y unidad de esfuerzo para cada análisis, utilizando también la extensión Animal Movement de ArcView 3.2 de ESRI (Hooge \& Eichenlaub, 2000).
Los datos sobre estimas de abundancia de delfín mular y calderón común Globicephala melas (Traill, 1809) se han extraído de trabajos realizados en el área de estudio y corresponden, en su totalidad, a estudios realizados por los autores de éste documento (Marcos et al., 2010, 2012).

\section{RESULTADOS}

\section{Esfuerzo de búsqueda y avistamientos}

A lo largo del periodo de estudio se han realizado 293 avistamientos de cetáceos, de los cuales 276 se han empleado para el análisis de datos. Se han identificado 12 especies de cetáceos, de las cuales cinco se han considerado habituales por su presencia continua a lo largo de la serie temporal y en cada una de las estaciones anuales (Fig. 2).

La especie más frecuente en el litoral guipuzcoano es el delfín mular, representando el $29 \%(n=81)$ de las observaciones, seguido por el delfín común $(28 \% ; n=$ $71)$, el calderón común $(18 \% ; n=49)$, el delfín listado Stenella coeruleoalba (Meyen, 1833$)(14 \% ; n=38)$ y el zifio de Cuvier Zifius cavirostris (Cuvier, 1823) (5\%; $n=$ 13). Todas ellas son especies consideradas residentes y/o habituales.

El cachalote Physeter macrocephalus (Linnaeus, $1758)$, con el $5 \%(n=13)$ de las observaciones, es la siguiente especie más observada en la costa guipuzcoana, seguida por el rorcual aliblanco Balaenoptera 

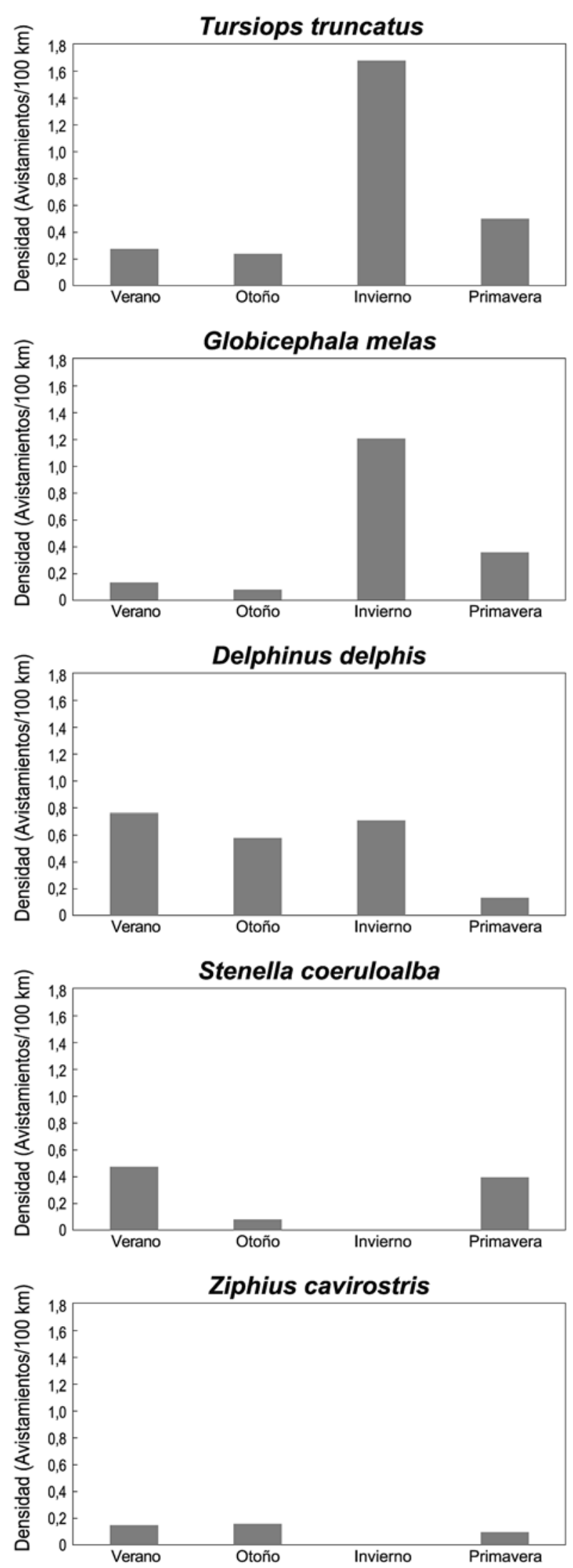

Fig. 2. - Distribución anual de las especies habituales de cetáceos en la costa guipuzcoana.

2. Irudia. Gipuzkoar kostaldeko ohiko ezpezieen urteroko banaketa. acutorostrata (Lacépède, 1804$)(2 \%, n=4)$ y por la marsopa Phocoena phocoena (Linnaeus, 1758) (1\%; $n=3)$.

El calderón de hocico boreal Hyperoodon ampullatus (Lacépède, 1804), otras especies de zifios Mesoplodon spp., el calderón gris Grampus griseus (Cuvier, 1812) y la yubarta Megaptera novaeangliae (Borowski, 1781) han sido observadas en una sola ocasión, representando cada una de estas especies el 0,4\% de los avistamientos totales.

\section{Distribución espacio temporal de las especies resi- dentes y/o habituales}

El delfín común se distribuye ampliamente y a lo largo de todo el año en la costa guipuzcoana (Fig. 2), tanto sobre la plataforma continental como en aguas oceánicas, mostrando preferencia por las aguas sobre zonas profundas de la fosa oceánica (Fig. 3).

El delfín listado se distribuye principalmente en aguas oceánicas, sobre profundidades de más de 200 m (Fig. 4). Principalmente ha sido detectado en verano, siendo en primavera y verano cuando mayor ha sido su densidad relativa en el área (Fig. 2).

El calderón común se ha observado en el área durante todo el año, siendo en invierno cuando mayor ha sido su tasa de encuentro (Fig. 2). Esta especie limita su área de distribución al talud y a las zonas profundas de la fosa oceánica (Fig. 5). Se ha estimado su población en 1.737 individuos (IC 95\%: 1.236-2.514 individuos) (Marcos et al., 2012).

El zifio de Cuvier concentra su área de acción en la zona más profunda del área estudiada (Fig. 6). Su densidad abarca un rango de 0,04 a 0,37 grupos $/ \mathrm{km}^{2}$. Esta especie se ha observado principalmente en primavera y verano (Fig. 2).

El delfín mular ha sido observado durante todo el año, siendo en invierno cuando mayor ha sido la densidad relativa de esta especie, con una tasa de encuentro de 0,02 grupos/km (Fig. 2). Esta especie se distribuye por toda la costa vasca, siendo la zona de la cabecera del cañón de Cap Breton su área de acción principal (Fig. 7). En una primera aproximación su población ha sido estimada en 1.579 individuos (IC 95\%: 918-2.830 individuos) (Marcos et al., 2010).

\section{Diversidad en aguas adyacentes a Jaizkibel}

El delfín mular y el delfín común campan habitualmente en el entorno marino de Jaizkibel, entendiendo como tal los $10 \mathrm{~km}$ contiguos al islote de Amuitz y la Bocana de la bahía de Pasajes (Fig. 3 y 7). En ocasiones se han detectado pequeños grupos adentrándose en las bahías de Pasaia y Txingudi. Así mismo, se han observado 3 especies de misticetos alimentándose en áreas adyacentes a la costa de Jaizkibel, como demuestran los 4 avistamientos de rorcual aliblanco, 3 de rorcual común y uno de yubarta realizados en la zona. 


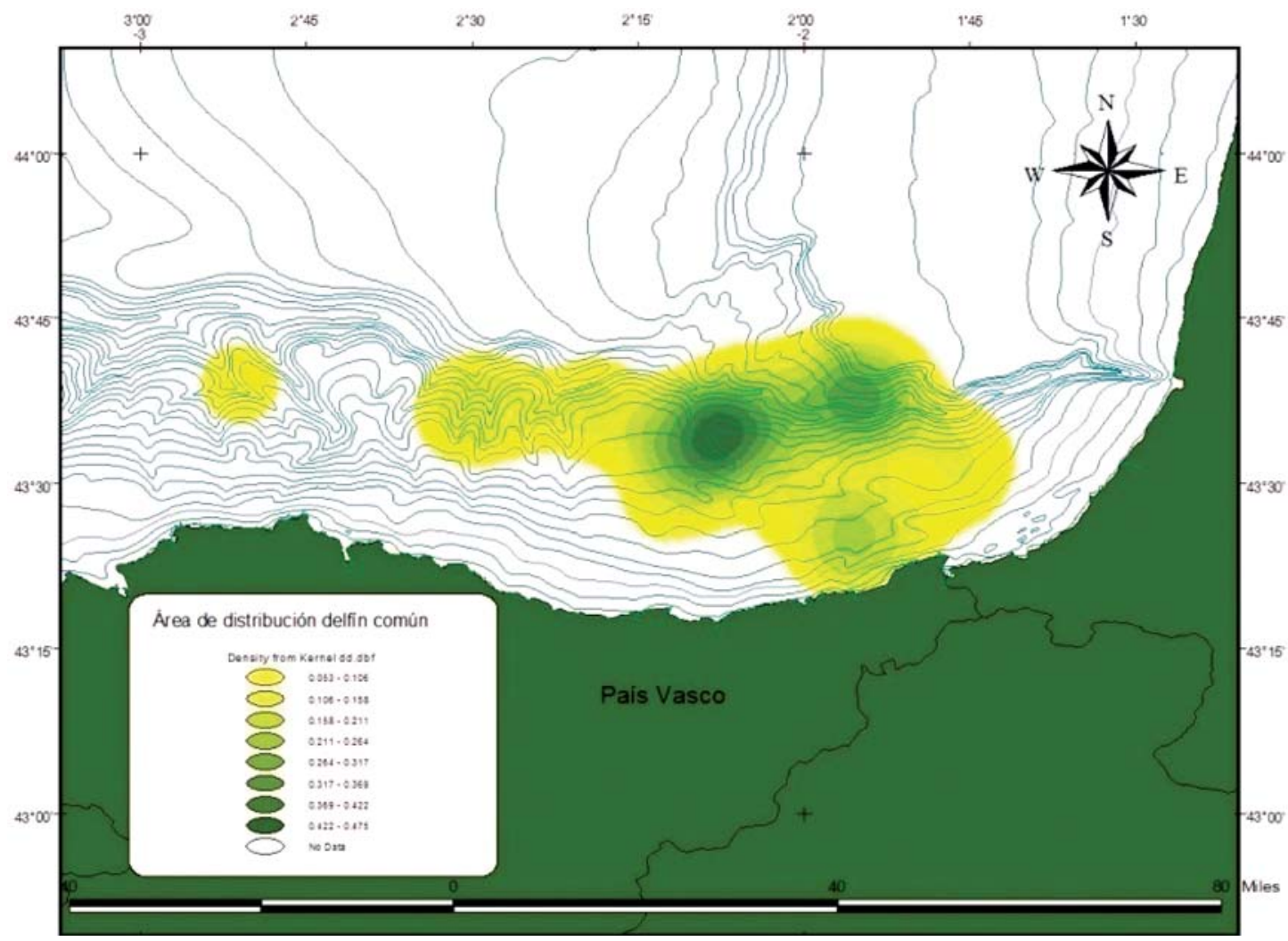

Fig. 3. - Distribución espacial del delfín común en la costa vasca oriental.

3. Irudia. Izurde arruntaren distribuzio ezpaziala ekialdetar euskal kostaldean.

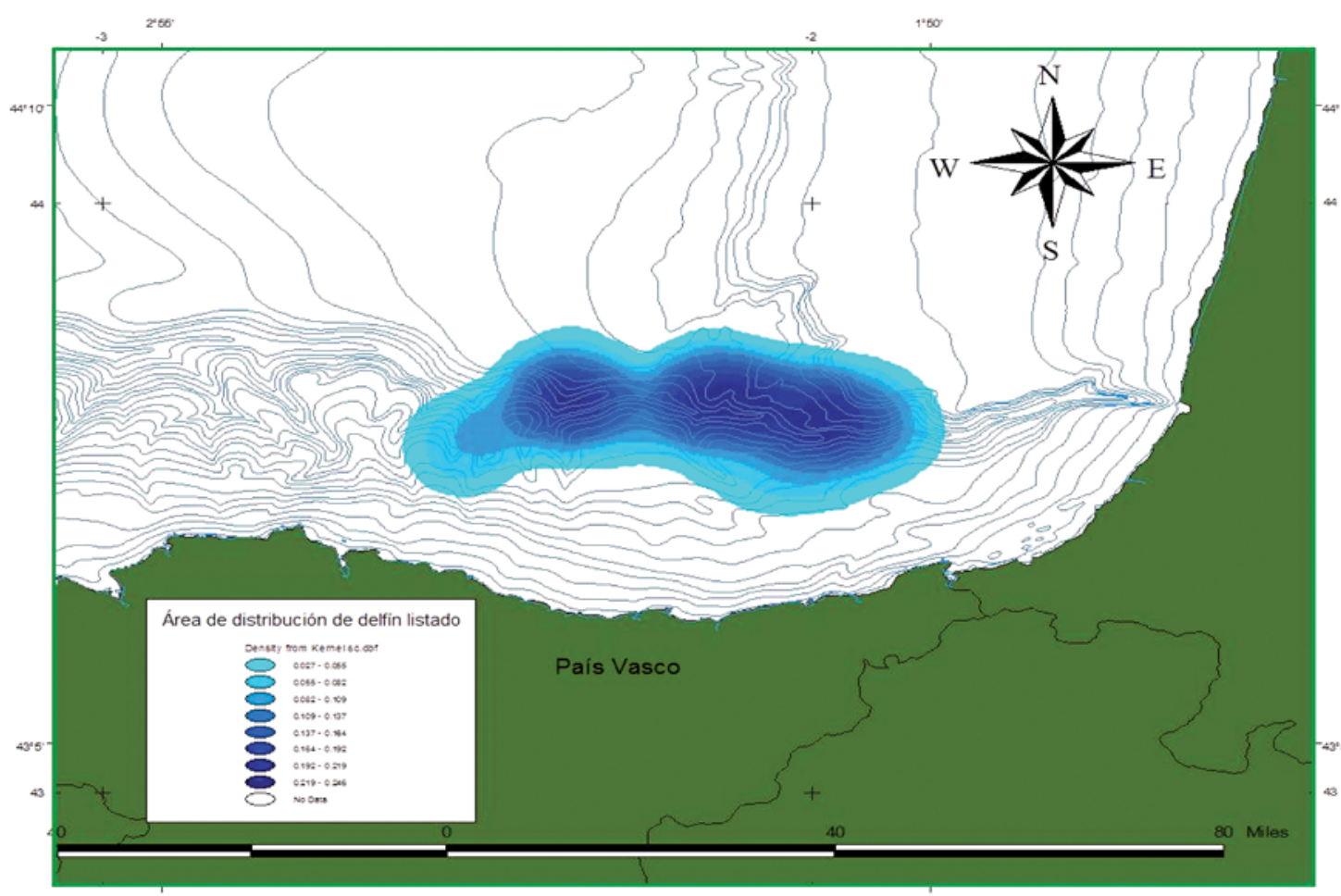

Fig. 4. - Distribución espacial del delfín listado en la costa vasca oriental. 4. Irudia. Izurde marradunaren distribuzio ezpaziala ekialdetar euskal kostal- 


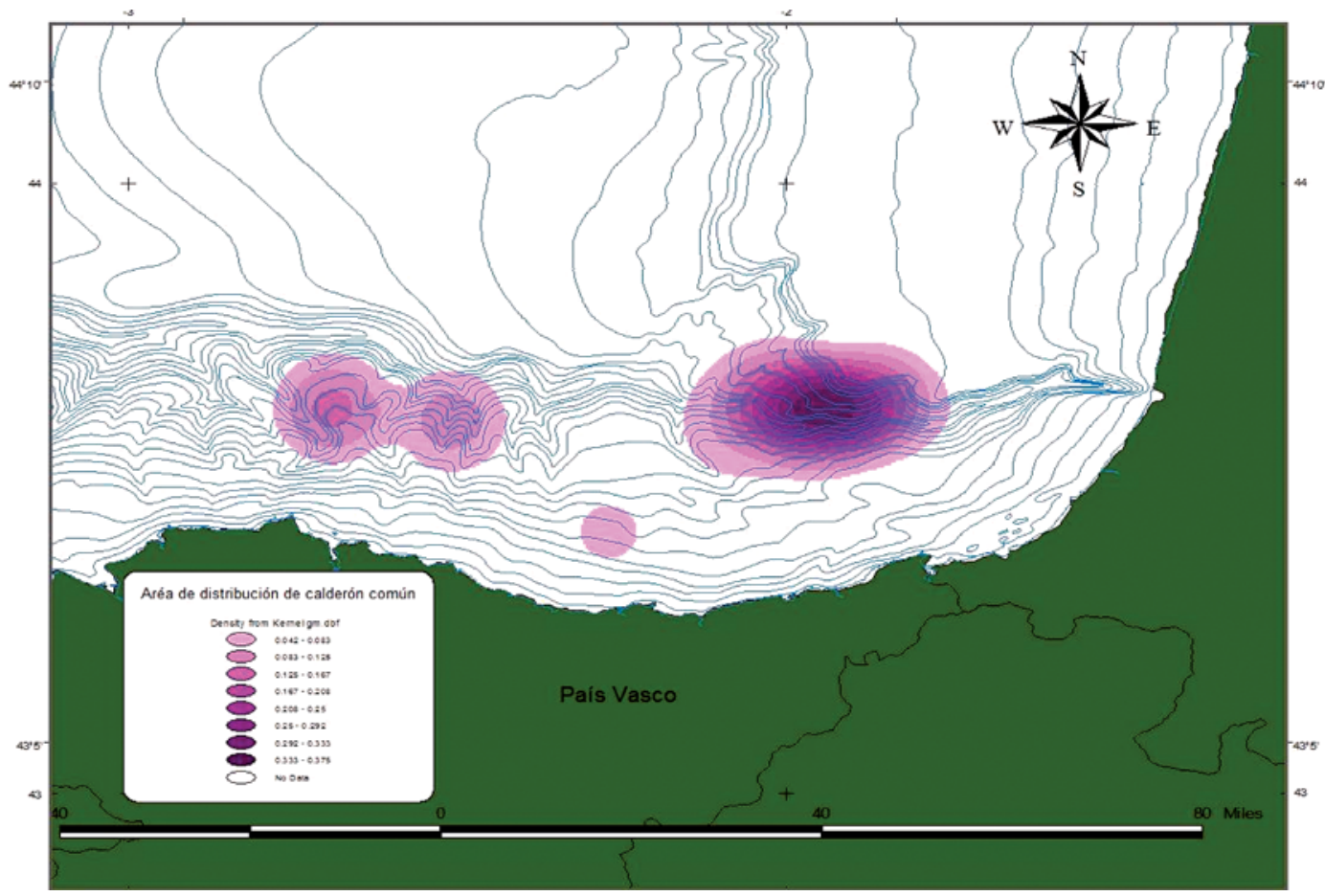

Fig. 5. - Distribución espacial del calderón común en la costa vasca oriental.

5. Irudia. Izurde pilotu hegats-luzearen distribuzio ezpaziala ekialdetar euskal kostaldean.

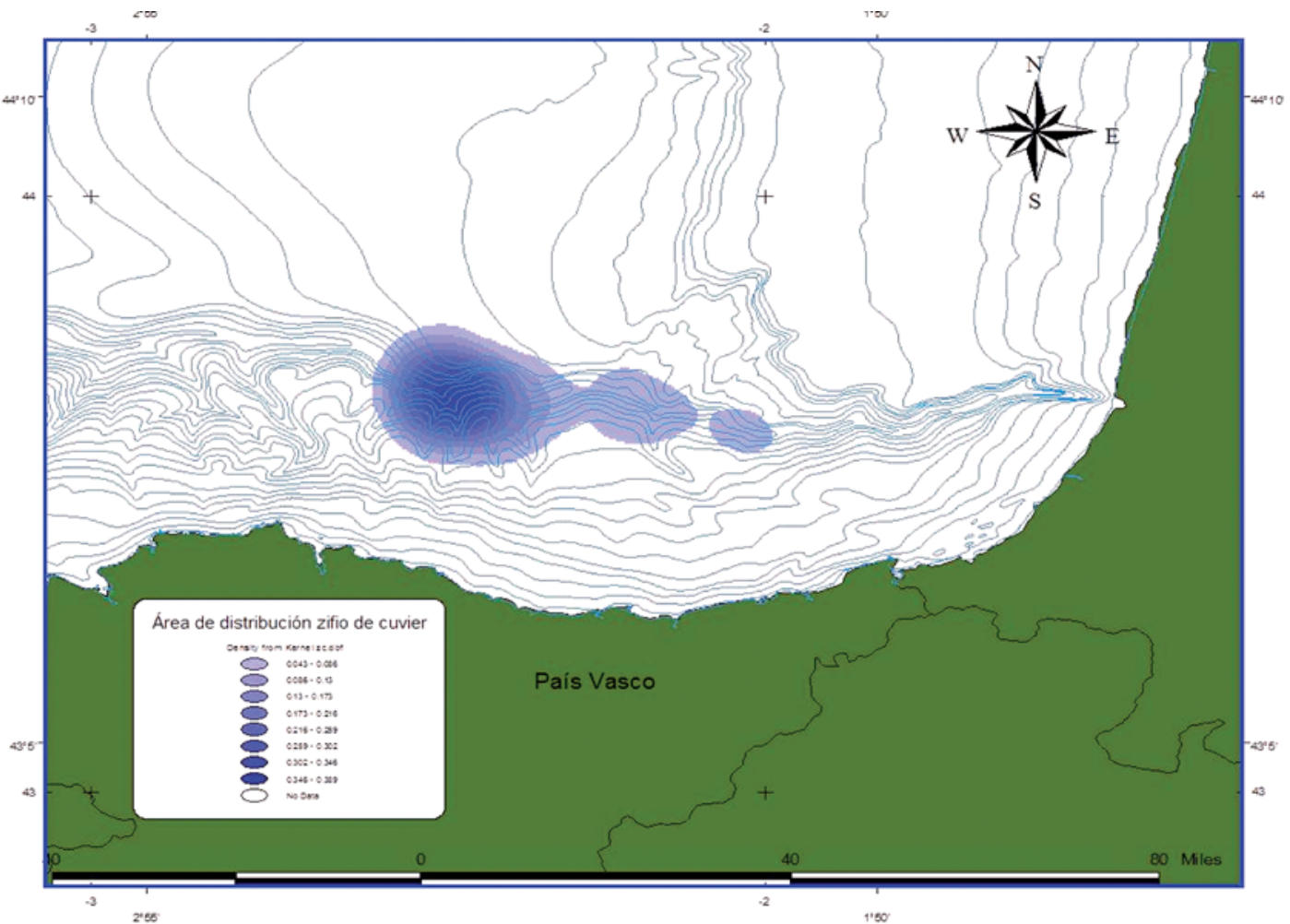

Fig. 6. - Distribución espacial del zifio de cuvier en la costa vasca oriental.

6. Irudia. Cuvier zifioaren distribuzio ezpaziala ekialdetar euskal kostaldean. 


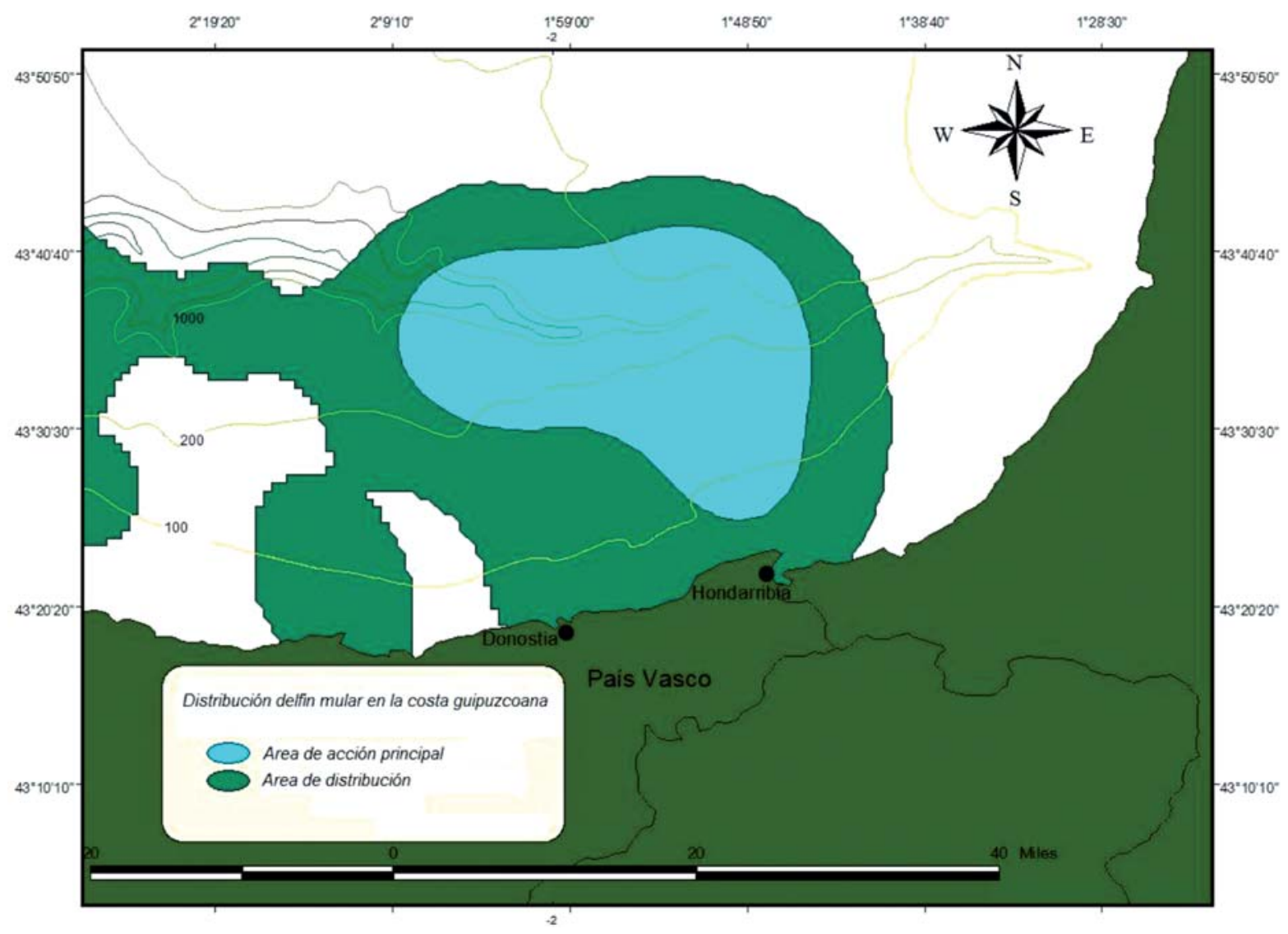

Fig. 7. - Distribución espacial y área de acción principal del delfín mular en la costa vasca oriental.

7. Irudia. Izurde haundiaren distribuzio ezpaziala eta banaketa eremu printzipalak ekialdetar euskal kostaldean.

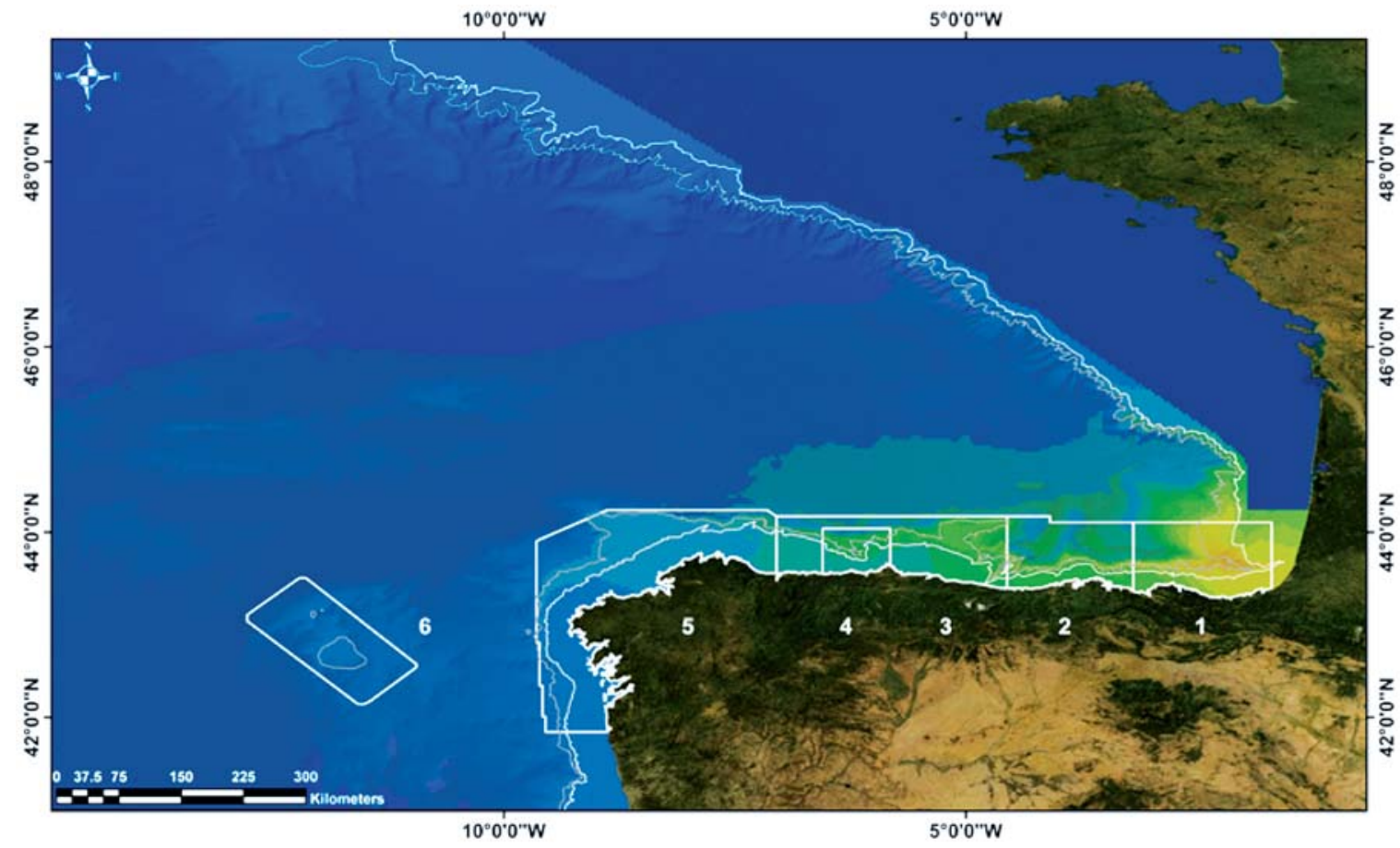

Fig. 8. - Densidad de delfín mular en la Demarcación Marina Noratlantica 2003-2011 (CEMMA, 2012)

8. Irudia. Izurde haundiaren dentsitate mapa Ipar Atlantikoko Demarkazio Itsastarrean 2003-2011 (CEMMA, 2012). 


\section{DISCUSIÓN}

En conjunto se detectan un total de 13 especies de cetáceos, lo cual supone el $81 \%$ de las especies registradas en la costa vasca (Marcos et al., 2013) y en todo el Atlántico Peninsular (Penas-Patiño, 1989; Aguilar, 1997; Ruano, 2007; López, 2013), el 65\% de las especies observadas en el Golfo de Bizkaia (Brereton, 2001; Walker, 2005b; Salazar, 2006, 2007; Laborde, 2008) y el 38\% de las especies observadas en aguas del Nordeste Atlántico (OSPAR, 2000). Teniendo en cuenta la reducida extensión del área y sin perder la referencia de la diversidad cetológica de toda la costa vasca, se han observado más especies que en áreas cercanas de dimensiones similares, como la costa asturiana y la cántabra, donde se han identificado 11 especies (Aguilar, 1997; Ruano, 2007). Podríamos concluir, en consecuencia, que la costa guipuzcoana es un área de interés para los cetáceos, que acoge más especies que las aguas más occidentales del Cantábrico, probablemente debido a la disponibilidad trófica de sus principales presas.

Entre las especies más frecuentes detectadas en la costa guipuzcoana, el delfín mular, el delfín común, el delfín listado, el calderón común y el zifio de Cuvier se han observado a lo largo de toda la serie temporal, por lo que se han considerado habituales. Esto supone que la costa de Gipuzkoa acoge de manera permanente al menos cinco especies de cetáceos (Marcos et al., 2010). El grado de fidelidad al área de estudio se conocerá mejor en futuros análisis sobre la estructura y la dinámica poblacional de cada una de las especies.

En cuanto al entorno marino más próximo a Jaizkibel, se confirma que es parte del área de distribución del delfín mular y del delfín común, donde también se ha registrado la presencia de misticetos alimentándose frente a Jaizkibel y la bocana de Pasajes. No obstante, se requiere mayor esfuerzo de investigación para determinar el uso del área por parte de estas especies, si bien se pone de manifiesto el interés de estas aguas para su conservación. Esto podría ser debido a la abundancia de presas potenciales para las distintas especies de cetáceos, promovida por la gran variedad de hábitats representados desde las aguas más someras a las de mayor profundidad.

Cabe destacar que el delfín mular es la especie más frecuente en el litoral guipuzcoano, y en las aguas más próximas a Jaizkibel en particular, siendo su principal área de campeo la cabecera del Cañón de Cap Breton, donde la densidad poblacional es de 0,39 grupos $/ \mathrm{km}^{2}$. En la costa de Jaizkibel esta densidad disminuye $\left(0,05\right.$ grupos $\left./ \mathrm{km}^{2}\right)$ (Marcos et al., 2010). Según los modelos predictivos obtenidos en el estudio coordinado por CEMMA (2012) sobre distribución y abundancia del delfín mular en el norte de la Península lbérica, se confirman las principales áreas de acción de la especie en la costa guipuzcoana, donde según López (2013) ha estimado la población en 1.931 individuos, siendo mayor que en las demás áreas estudiadas (Cantabria: 744, Asturias: 1.214, Galicia: 703).

Todas estas especies, así como otras citadas para la costa vasca (Marcos et al., 2005, 2013; Vázquez et al.,
2005), están incluidas en los anexos II y IV de la Directiva Hábitat (92/43/CEE), según la cual se requiere determinar las áreas de máximo interés para su conservación, profundizar en su estudio y desarrollar un programa de gestión que asegure su protección. En el caso del delfín mular y la marsopa, ambas presentes en el área de estudio (Marcos et al., 2010) e incluidas en el anexo II, es necesario, además, delimitar Zonas Especiales de Conservación en territorio europeo. En este contexto, se pone de manifiesto la necesidad de crear un Área Marina Protegida para asegurar su protección y cumplir con las directrices de la Directiva Hábitat y la Estrategia Española para la Conservación y el Uso Sostenible de la Diversidad Biológica (DGCN, 1998).

\section{BIBLIOGRAFÍA}

Aguilar, A., Forcada, J., Arderiu, A., Borrel, A., Monna, A., Aranburu, M.J., Pastor, T., Cantos, G. 1997. Inventario de los cetáceos de las aguas atlánticas Peninsulares: aplicación de la directiva 92/43/CEE. Universitat de Barcelona, Departamento de Biología Animal.

Anderwald, P., Evans, P.G.H. 2007 Minke Whale Populations in the North Atlantic an Overview with Special Reference to UK waters. European Cetacean Society. Spec. Public. Series 47: 8-13.

Arregi, L., Puente, E., Lucio, P., Sagarminaga,Y., Castro,R.,Uriarte A. 2004. Coastal fisheries and Demersal Fauna. En: Oceanography and Marine Environment of the Basque Country. A. Borja, M. Collins (Ed.): 493-514. Elsevier. Amsterdam.

Borja, A., Aguirrezabalaga, F., Martínez J., Sola, J.C., García-Arberas, L., Gorostiaga, J.M. 2004. Benthic communities, biogeography and resources managment. En: Oceanography and Marine Environment of the Basque Country. A. Borja, M. Collins, (Ed.): 455-492. Elsevier. Amsterdam.

Borja, A., Collins, M. 2004. Oceanography and Marine Environment of the Basque Country. Elservier. Amsterdam. (Elsevier Oceanography Series; 70).

Brereton, T., Wall, D., Cermeño, P., Vazquez, J.A., Curtis, D., Williams A. 2001. Cetacean monitoring in North-West European waters. Atlantic Research Coalition (ARC). (Report Number; 1)

Casamajor, M.N. de. 2004. Baie de Biscaye, richesse méconnue \& diversité. Ed. Alexandre Dedwez. Ascain.

Catálogo Nacional de Especies Amenazadas (R.D. 439/1990) Disponible en web: http://www.magrama.gob.es /es/biodiversidad/temas/conservacion-de-especies-amenazadas/catalogonacional-de-especies-amenazadas /acceso_catalogo.aspx.

CEMMA. 2012. Bases para el desarrollo de los planes de conservación de las especies de cetáceos protegidas en la "Demarcación Marina Noratlántica. Propuesta de Planes de Conservación Demarcación Marina Noratlántica. Ministerio de Agricultura, Alimentación y Medio Ambiente. Pendiente publicación.

DGCN. 1998. Estrategia Española para la conservación y el uso sostenible de la diversidad biológica. Ministerio de Medio Ambiente, Secretaría General de Medio Ambiente. Madrid.

Directiva 92/43/CEE, relativa a la conservación de los hábitats naturales y de fauna y flora silvestres. Disponible en web: http://www.ingurumena.ejgv.euskadi.net.

EIBE. 2010. Estudio piloto para la realización de una propuesta para la definición de Lugares de Interés Comunitario en el litoral de la Comunidad Autónoma del País Vasco. Gobierno Vasco, Departamento de Medio Ambiente, Planificación Territorial, Agricultura y Pesca. Vitoria-Gasteiz. 
Hoyt E. 2005. Marine Protected Areas for whales, dolphins and porpoises: a world handbook for cetacean habitat conservation. Earthscan. London.

Hooge, P.N., Eichenlaub, B. 2000. Animal movement extension to Arcview, ver 2.0. Alaska Science Center - Biological Science Office, U.S. Geological Survey. Anchorage, AK, USA. Disponible en web: http://www.absc.usgs.gov/glba/gistools/ index.htm\#ANIMAL MOVEMENT.

Laborde Basto d'Andrade, M.I. 2008. Spatial distribution of cetaceans in the Bay of Biscay and implications of the Marine Strategy Directive for their conservation. Universidade de Lisboa, Departamento de biología animal. Master Tesis.

Lens, S., Santos, M. B., Oñate, D., Miranda, A., Casas, G., Cañadas, A Cabanas, J M. Iglesias, M. Miquel, J., Fernández, R. Vázquez, J. A. 2008. Distribution of fin whales and krill aggregations observed off the galician coasts during the CODA-IEO survey. SYMPOSIUM The Role of Marine Mammals in the Ecosystem in the 21st Century 29 Sept- 1 Oct. Canada.

López, A., Covelo, P., Vázquez, J.A., Mártinez, J.A., Caldas, M., Lago, R., Alonso J.M. 2007. Bases para la conservación y gestión de las especies de cetáceos amenazadas en las aguas atlánticas y cantábricas. Informe de Proyecto, Coordinadora para o Estudio dos Mamíferos Mariños (CEMMA)-Fundación Biodiversidad

López, A., Vázquez, J.A., Cedeira, J., Cañadas, A, Marcos, E. Maestre, I., Ruano, A., Laria L., Hammond, P., Macleod K., Evans, P. 2013. Conferencia Annual de la European Cetacean Society, Setubal, Portugal, 8-10 Abril 2013. New abundance estimates of harbour porpoise (Phocoena phocoena) and bottlenose dolphin (Tursiops truncatus) in Northern Spanish Cantabrian and adjacent waters of Bay of Biscay (2003-2011). Poster 27.

Marcos, E, Salazar-Sierra, J.M., Stephanis, R. de. 2005. Proceedings of the 19 Conferencia Annual de la European European Cetacean Society, La Rochelle, Francia, 2-7 Abril 2005. Cetacean population in coast of the Basque Country: Diversity and distribution spring-summer 2003-2004. (European Research on cetaceans; 19).

Marcos, E., Salazar, J.M., Stephanis, R. de. 2010. Cetacean diversity and distribution in the coast of Gipuzkoa and adjacent waters, southeastern Bay of Biscay. Munibe, Cienc. Nat. 58: 221-231.

Marcos, E., Martinez-Jorquera, J., Verborgh, P., Salazar, J.M. 2012. 26th Annual Conference of the European Cetacean Society, Galway, Irlanda, 26-28 Marzo 2012. Long-finned pilot whale population size and temporal density fluctuations in Basque Coast, Southeastern Bay of Biscay.

Marcos, E., Salazar, J.M. 2013. VII Congreso de la Sociedad Española de Cetáceos. Tarifa, Cádiz, 4-6 octubre 2013. Estudio y conservación de las poblaciones de cetáceos en la costa vasca.

Nogeira, E., Batleb, J.M., Cabal J., Gonzalez-Nuevo, G., Revilla, R., Alvarez E., Bueno, J. 2008. Accumulation of northern krill (Meganyctiphanes norvegica) in a convergence zone at the Cap Breton Canyon (southern Bay of Biscay). Rev. Invest. Mar. 3: 225-226.

OSPAR. 2000. Quality Status Report 2000: Region IV - Bay of Biscay and Iberian Coast. OSPAR Commission. London.

Penas-Patiño, X.M., Piñeiro-Seage, A. 1989. Cetáceos, focas e tartarugas das costas ibericas. Santiago de Compostela: Conselleria de Pesca, (Xunta de Galicia). Fernandez de la Cigonña, E., 1990.Vol. III. Os cabaleiros do mar: baleas e golfiños das nosas augas. Guía dos cetáceos de Galicia e Iberia. En: Natureza Galega. Asociación Galega para a Cultura e a Ecoloxía (AGCE).

Powell, R.A. 2000. Animal home ranges and territories and home range estimators. In: Research techniques in animal ecology: controversies and consequences. L. Boitani, T. K. Fuller (Ed.): 65-110. Colombia University Press. New York.

Read, F.L., Santos, S., González, A.F., Martínez-Cedeira, J., López, A., Pierce, G. J. 2008. 61st Annual Meeting of the International Whaling Commission. Madeira, Portugal 1-13 June 2009.
Common dolphin (Delphinus delphis) in Galicia, NW Spain: dis tribution, abundance, life history and conservation (SC/61/SM5).

Ruano A., Silva, P., Solano, S., Naves, J. 2007. Cetáceos del litoral asturiano: áreas de interés para la conservación. La Caixa.

Salazar-Sierra J.M., Marcos-Ipiña E. 2007. 21th Annual Conference of the European Cetacean Society, Donostia-San Sebastian, Spain, April 2007. Mysticeti populations in the bay of biscay: Diversity and distribution (summer-autumn 2003-2004-2005).

Salazar-Sierra, J.M., Marcos-Ipiña, E. 2006. Proceedings of the 20th Annual Conference of the European Cetacean Society, Gdynia, Poland, 2-7 April 2006. Odontocete populations in the Bay of Biscay: Diversity and distribution (summer-autumn 2003-20042005). (European Research on cetaceans; 20).

Santos Vazquez, M.B., Fernandez, R., Lopez, A., Martinez, J.A Pierce, G.J. 2007. Variability in the diet of bottlenose dolphin, Tursiops truncatus in Galician waters, north-western Spain, 19902005. J. Mar. Biol. Assoc. U. K. 87(1): 231-241.

Seaman, D.E., Powell, R.A. 1996. An evaluation of the accuracy of kernel density estimators for home range analysis. Ecology 77 2075-2085.

Seaman, D.E., Millspaugh, J.J., Kernohan, B.J., Brundige, G.C., Raedeke, K.J., Gitzen, R.A. 1999. Effects of sample size on kernel home range estimates. J. Wildl. Manag. 63: 739-747.

SEC. 1999. Recopilación, Análisis, Valoración y Elaboración de Protocolos sobre las labores de observación, asistencia a varamientos y recuperación de mamíferos y tortugas marinas de las Aguas Españolas. Technical Report. Sociedad Española de Cetáceos

Spitz J., Richard, E., Meynier, L., Pusineri, C., Ridoux, V. 2006 Dietary plasticity of the oceanic striped dolphin, Stenella coeruleoalba, in the neritic waters of the Bay of Biscay. J. Sea Res. 55: 309-320

Spitz, J., Cherel, Y., Bertin, S., Kiszka, J., Dewez, A., Ridoux, V 2011. Prey preferences among the community of deep-diving odontocetes from the bay of biscay, northeast atlantic. Deep-Sea Research Part I. Oceanogr. Res. Pap. 58(3): 273-282.

Valencia, V., Franco, J., Borja, A., Fontán, A. 2004. Hydrography of the southeastern Bay of Biscay. En: Oceanography and Marine Environment of the Basque Country. A. Borja, M. Collins (Ed.): 159-194. Elsevier. Amsterdam.

Vázquez. J.A. 2005. Distribución y uso de hábitat del delfín mular (Tursiops truncatus), calderón de aleta larga (Globicephala melas) y de zifio común (Ziphius cavirostris), en aguas cercanas a la costa vasca. Gobierno Vasco. Departamento de medio ambiente y ordenación del territorio Biodiversidad.

Walker, D., 2005a. Using oceanographic features to predict areas of high cetacean diversity. MSc Thesis. University of Wales. Bangor.

Walker, D., McHenry, M., Hickey, R., Clemente, S., Beaumont N.,Macleod, K. 2005b. Mapping Cetacean Biodiversity in the Bay of Biscay. Poster presentation. Organisation Cetacea, UK

Worton, B.J. 1995. Using Monte Carlo simulation to evaluate kernel based home range estimators. J. Wildl. Manag. 59: 794-800. 